\title{
西伯利亚鲟仔鱼侧线系统的发育
}

\author{
宋 炜 $1,2,3$, 宋佳坤 ${ }^{1,2, *}$ \\ （1. 上海海洋大学 海洋生物系统和神经科学研究所, 上海 201306; 2. 上海海洋大学 水产与生命学院, 上海 201306; 3. 农业部东海与远洋渔业 \\ 资源开发利用重点实验室，中国水产科学研究院 东海水产研究所，上海 200090)
}

摘要: 鲟鱼属软骨硬鳞鱼, 在电感受器的进化中占据着极为重要的地位。该文以光镜和扫描电镜手段研究了 西伯利亚鲟侧线系统早期发育, 包括侧线基板发育及感觉崉的形成、侧线感受器的发育和侧线管道的形成。1 日 龄, 听囊前后外胚层增厚区域出现 6 对侧线基板; 除后侧线基板细胞向躯侧面迁移外, 其他侧线基板均形成感 觉嵴结构; 每一侧线基板中均有神经丘原基形成。7 日龄, 壶腹器官在吻部腹面两侧出现, 壶腹器官的发育比神经 丘晚一周左右。9 日龄，神经丘下的表皮略有凹陷，侧线管道开始形成。29日龄，在吻部腹面两侧可见少数个别的 壶腹器官表皮细胞覆盖壶腹器官中央区域留下 $3 \sim 4$ 个小的开口; 壶腹管内可见大量的微线毛存在, 在其他鲟形目 鱼类、软骨鱼类中也存在类似的结构。57 日龄, 躯侧线管道己完全埋于侧骨板中; 壶腹器官主要分布在吻部腹 面, 3 4 个聚集在一起，呈“梅花状”，分布紧密，并且该部分皮肤表面凹陷，形成花朵状凹穴; 侧线系统发育完善。

关键词: 鲟鱼; 神经丘; 壶腹器官; 电感受器; 侧线基板

中图分类号：Q959.463; Q432＼cjkstart文献标志码：A＼cjkstart文章编号：0254-5853-(2012)03-0261-10

\section{Development of the lateral line system in juvenile Siberian sturgeon (Acipenser baerii)}

\author{
SONG Wei ${ }^{1,2,3}$, SONG Jia-Kun ${ }^{1,2, *}$ \\ (1. Institute for Marine Biosystem and Neurosciences, Shanghai Ocean University, Shanghai 201306, China; 2. College of Fishery and Life Science, Shanghai \\ Ocean University, Shanghai 201306, China; 3. Key Laboratory of East China Sea and Oceanic Fishery Resources Exploitation, Ministry of Agriculture; East \\ China Sea Fisheries Research Institute, the Chinese Academy of Fishery Science, Shanghai 200090, China)
}

\begin{abstract}
The Siberian sturgeon (Acipenser baerii Brandt), a chondrostean, occupies an important position in the evolution of the electroreceptor. In order to more fully understanding the evolution of these receptors, we examined the development of the lateral line system during early ontogeny of the Siberian sturgeon by using light and scanning electron microscopes. We detected four major events in this process: the lateral line placodal development, the sensory ridge formation, the receptor formation and the canal formation. On day 1 of post hatching, all six lateral line placodes are present and the posterior lateral line placode starts actively migrating posteriorly along the mid-line of the trunk, depositing neuromasts at intervals on the way of migration. The other lateral line placodes elongate to form sensory ridges according to its destination line pattern over the head, all containing primordial neuromasts. By day 7, ampullary organs rise from the lateral zones of the ventral of the head, though this may lag up to one week behind of that of the neuromasts. On day 9, the epidermis under the neuromast slowly invaginates, and the bony lateral line canals begin to form. Towards day 29, the epidermal cells surrounded some single openings of the ampullary organs at the ventral surface of the head, begin to migrate, and then transform into 3 to 4 aggregate openings. By this point, abundant microvilli are visible on the surface of the receptor epithelium, similar to the structure in elasmobranches and other sturgeons. On the day 57 of post hatching, the trunk canal is fully embedded into the lateral scutes. By then, the majority of ampullary organs are highly concentrated on the ventral rostrum, arranged in clusters of $3-4$, distributing closely such as the shape of quincunx, thus completing the formation of the lateral line system.
\end{abstract}

Key words: Sturgeon; Neuromasts; Ampullary organs; Electroreceptors; Lateral line placodes

收稿日期: 2012-01-16; 接受日期: 2012-03-24

基金项目: 上海市科学技术委员会 2008 年科研计划资助项目(073205109); 公益性行业科研专项经费项目(201003055-07)

通信作者(Corresponding author), 教授, 博士生导师, E-mail: jksong@shou.edu.cn

第一作者简介: 宋炜(1983一), 男, 助理研究员, 博士, 研究方向: 鱼类形态及发育生物学研究。E-mail: swift83@sina.com 
侧线系统是皮肤衍生的感觉器官, 为鱼类和水 生两栖类所特有, 在鱼类的摄食、御敌、生殖、洄 游等行为中发挥重要作用(Baker \& Bronner-Fraser, 2001; Goulet et al, 2008; Mogdans \& Geisen, 2009; Wilkens \& Hofmann, 2007)。它由机械感受器和电感 受器组成。机械感受器能感受水流、水压的变化; 电感受器能检测周边微弱电场变化(Cheng et al, 1995; Northcutt, 1992; Song et al, 1995)。不同于机械 感受器, 电感受器在硬骨鱼类中经历了二次进化, 如部分辐鯺亚纲鱼类(多鳍鱼和鲟鲌鱼等)存在与软 骨鱼类相似的壸腹型电感受器; 而新鯺亚纲中的雀 鱚目、弓鯺鱼目电感受器缺失, 仅存在机械感受器。 在部分现代真骨鱼(骨舌鱼类和个别鲶鱼等)中又出 现了结节状电感受器, 但电感受器的进化历程, 尚 未完全了解(Gibbs, 2004; O'Neill et al, 2007)。

鲟鱼是软骨硬鳞下纲中现存的唯一一目, 素有 “活化石”之称(Ma, 2007; Wang et al, 2009)。它是少 数具有壸腹型电感受器的硬骨鱼类之一, 在电感受 器进化中占据极为重要的地位 (Gibbs \& Northcutt, 2004; Hofmann et al, 2002; Song et al, 2010)。西伯利 亚鲟 (Acipenser baerii Brandt) 隶属鲟形目 (Acipenseriformes) 鲟科 (Acipenseridae) 鲟 属 (Acipenser), 主要分布于俄罗斯西部的鄂毕河至东 部的科雷马河之间的各河流中 (Gisbert \& Williot, 2002)。西伯利亚鲟头部具较为丰富的电感受器 (Gibert et al, 1999), 且适应性广、生长速度快、具有 抗病能力, 现已成为我国淡水养殖的优良品种(Lin, 2003)。近年来, 有关西伯利亚鲟的研究已有不少, 在早期发育方面, 对其早期行为学、眼睛发育已进 行了较系统的研究 (Gisbert \& Ruban, 2003; Rodríguez \& Gisbert, 2002), 而侧线系统发育相关 研究尚未见报道。本研究从形态学和组织学角度, 应用光镜和扫描电镜研究西伯利亚鲟侧线系统早 期发育, 不仅对研究电感受器的起源与进化有着重 要意义, 而且为人工育苗积累基础资料。

\section{1 材料与方法}

\section{1 实验材料}

西伯利亚鲟受精卵为杭州千岛湖鲟龙科技开 发有限公司全人工繁殖所得子二代 $\left(\mathrm{F}_{2}\right)$ 卵。受精卵在 实验室经人工孵化, 孵化温度控制在 $(18 \pm 0.7){ }^{\circ} \mathrm{C}$ 。 出膜后的仔鱼置于 $35 \mathrm{~cm}($ 长) $\times 21 \mathrm{~cm}$ (宽)的塑料水
族箱中饲养，水深保持 $15 \mathrm{~cm}$ 。实验用水需经充分 曝气, 用加热棒控温。开口饵料为水蚯蚓, 进入稚 鱼阶段后投喂颗粒饲料。实验过程中每天投喂 2 次, 排污和换水各 1 次, 每次换水量为水体的 $1 / 4$ 。

\section{2 实验方法}

记载鱼苗出膜高峰期当天为 0 日龄。早期仔鱼 阶段每 $24 \mathrm{~h}$ 取材 1 次; 晚期仔鱼和稚鱼阶段, 每 48 $\mathrm{h}$ 取材 1 次。每阶段取材 10 尾, 立即用 3-氨基苯甲 酸乙酯甲基磺酸盐(MS-222)麻醉, 其中 5 尾经 Bouin 氏液固定后，按照常规组织学方法制作石蜡 切片, 作连续切片(厚度 5 7 $\mu \mathrm{m}$ ), H.E. 染色, 中性 树胶封片, Olympus 显微镜下观察并拍照。另外 5 尾, 经 $2.5 \%$ 戊二醛和 $1 \%$ 锇酸双重固定, 梯度酒 精脱水，醋酸异戊酯取代，临界点干燥，喷金， JEOL-6390LV 扫描电镜观察并拍照。

\section{2 结果与分析}

西伯利亚鲟侧线系统早期发育过程主要包括 侧线基板发育及感觉嵴的形成、侧线感受器的发育 以及侧线管道的形成。

\section{1 侧线基板发育及感觉嵴的形成}

1 日龄, 刚出膜的仔鱼头部较小, 弯向卵黄囊; 嗅囊呈椭圆形, 触须原基和鳃原基形成(图 1A)。在 听囊前后外胚层增厚区域出现 6 对侧线基板。听囊 前有 3 对分布，分别为前背侧基板、前腹侧基板、 听侧线基板; 听囊后的 3 对分别为中间基板、横枕 基板和后侧线基板(图 1B)。侧线基板成神经细胞形 成(图 2A, B)。前背侧基板、前腹侧基板、听侧线基 板、中间基板、横枕基板开始延长形成过渡性结构 一感觉嵴, 神经丘原基在所有的感觉嵴上形成, 并 且在听侧线基板感觉嵴中央区域已有 3 个神经丘出 现(图 2C); 后侧线基板没有形成感觉嵴结构, 而是 开始向躯干部迁移，在躯干部特定的位置遗留下部 分细胞, 这群细胞最终将发育成为神经丘(图 2D)。

5 日龄，仔鱼头部抬起并伸长，嗅囊中间内侧 靠拢，呈“哑铃状”，鳃丝外露，触须增长，呈圆柱状 (图 1C)。前背侧基板感觉嵴分别向睈上和睈下延伸, 形成眀上感觉嵴和眀下感觉嵴。睈上感觉嵴出现 4 个神经丘; 眶下感觉嵴出现 1 个神经丘。前腹侧 基板感觉嵴向背部略有延长。听侧线基板感觉嵴 和中间感觉嵴在同一直线上，听侧线基板出现 4 个 神经丘, 中间基板出现 1 个神经丘; 横枕感觉嵴向 

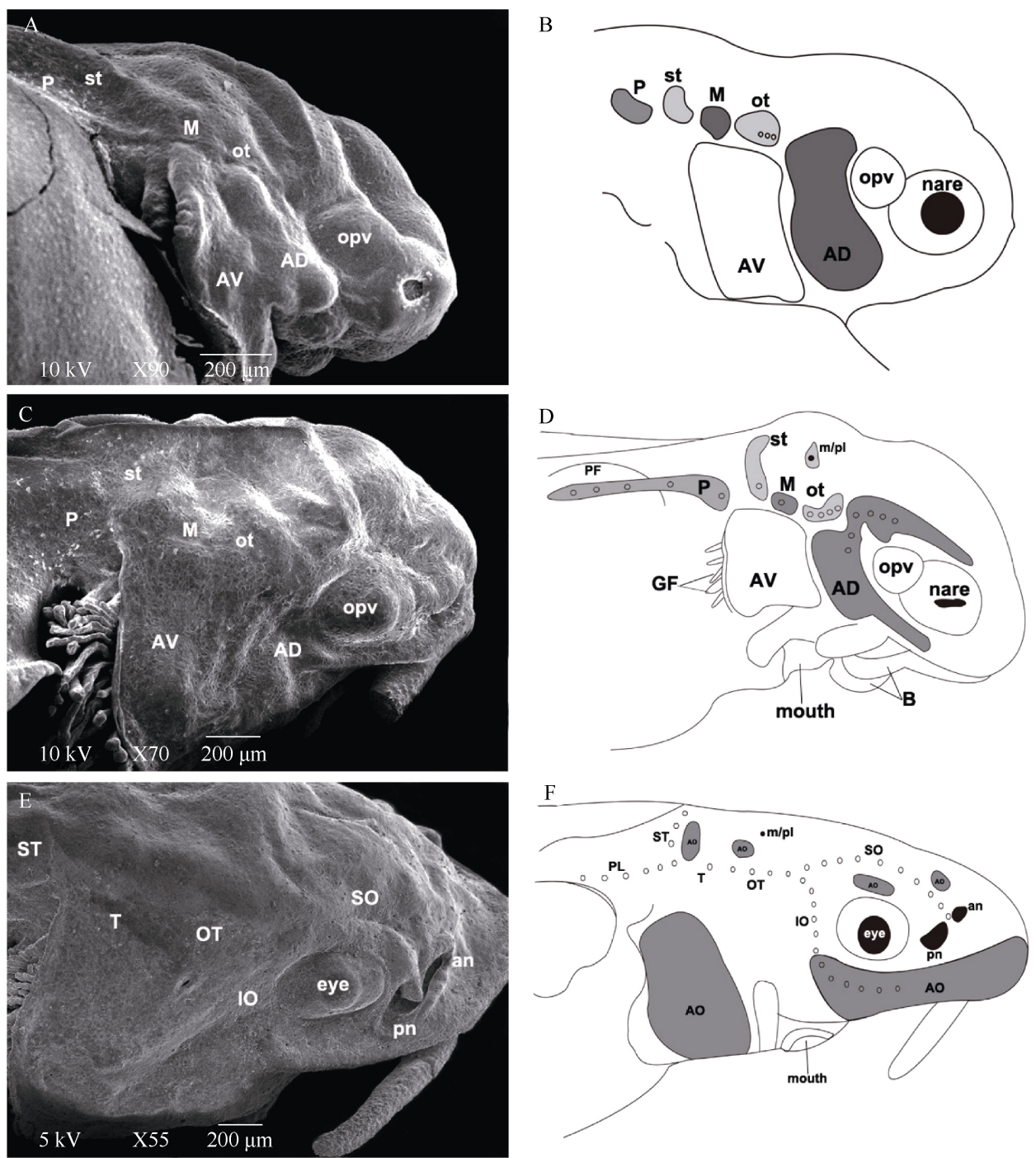

图 1 西伯利亚鲟头部扫描电镜图(A, C, E)及相关侧线发育模式图 $(\mathrm{B}, \mathrm{D}, \mathrm{F})$

Fig.1 Scanning electron micrographs of the lateral surface of the head (A,C,E) and the camera lucida drawings of the lateral view of the head $(\mathrm{B}, \mathrm{D}, \mathrm{F})$

A, B) 1 日龄仔鱼头部侧面; C, D) 5 日龄仔鱼头部侧面; E, F) 9 日龄仔鱼头部侧面。AD: 前背侧基板; AV: 前腹侧基板; ot: 听侧线基板; M: 中间基板; st: 横枕基板; P: 后侧线基板; opv: 视泡; $\mathrm{m} / \mathrm{pl}$ : 中间或后陷器线; B: 触须; PF: 胸鯺; GF: 鳃丝; SO: 眶上线; IO: 眶下线; $\mathrm{OT}$ : 听侧线; $\mathrm{T}$ : 颢线; $\mathrm{ST}$ : 横枕线; PL: 后侧线; an: 前鼻孔; pn: 后鼻孔; AO: 壸腹器官(深灰色阴影部位)。

A, B) day 1 post hatching; C, D) day 5 post hatching; E, F) day 9 post hatching. AD: anterodorsal placode; AV: anteroventral placode; ot: otic lateral line placode; M: middle placode; st: supratemporal placode; P: posterior placode; opv: optic vesicle; m/pl: middle/posterior pit lines; B: barbells; PF: pectoral fin; GF: gill filament; SO: supraorbital line; IO: infraorbital line; OT: otic lateral line; T: temporal lateral line; ST: supratemporal line; PL: posterior lateral line; an: anterior nare; pn: posterior nare; AO: ampullary organs (dark gray shaded portion).

背部的中线迁移, 出现 1 个神经丘; 后侧线基板已 迁移至胸鯺后端，并有 5 个神经丘形成; 此外，在 中间陷器线和后陷器线的位置有 1 个神经丘出现; 壶腹器官原基在前背侧基板中形成(图 1C, D)。

9 日龄, 仔鱼头部扁平, 鼻孔中间缝合, 形成前 后两鼻孔，口呈水平位(图 1E)，此时仔鱼开始营底 栖生活, 营养从内源向外源转换, 仔鱼各器官快速 发育。眶上感觉嵴和睈下感觉嵴的后端连接处已与
听侧线基板感觉嵴相连接。中间基板感觉嵴向前后 轴伸长, 前端与听基板感觉崉融合, 后端与横枕基 板感觉嵴、后侧线基板融合，此时侧线管道开始形 成。神经丘在所有感觉嵴中央出现, 其中眶上管 10 个, 眶下管 17 个, 听管 4 个, 囬管 2 个, 横枕管 3 个。 后侧线基板已迁移至尾部。壸腹器官在前背侧基 板、前腹侧基板、听基板、横枕基板中出现，并且在 前背侧基板中有少数壸腹器官发育完全(图 $1 \mathrm{E}, \mathrm{F}$ )。 

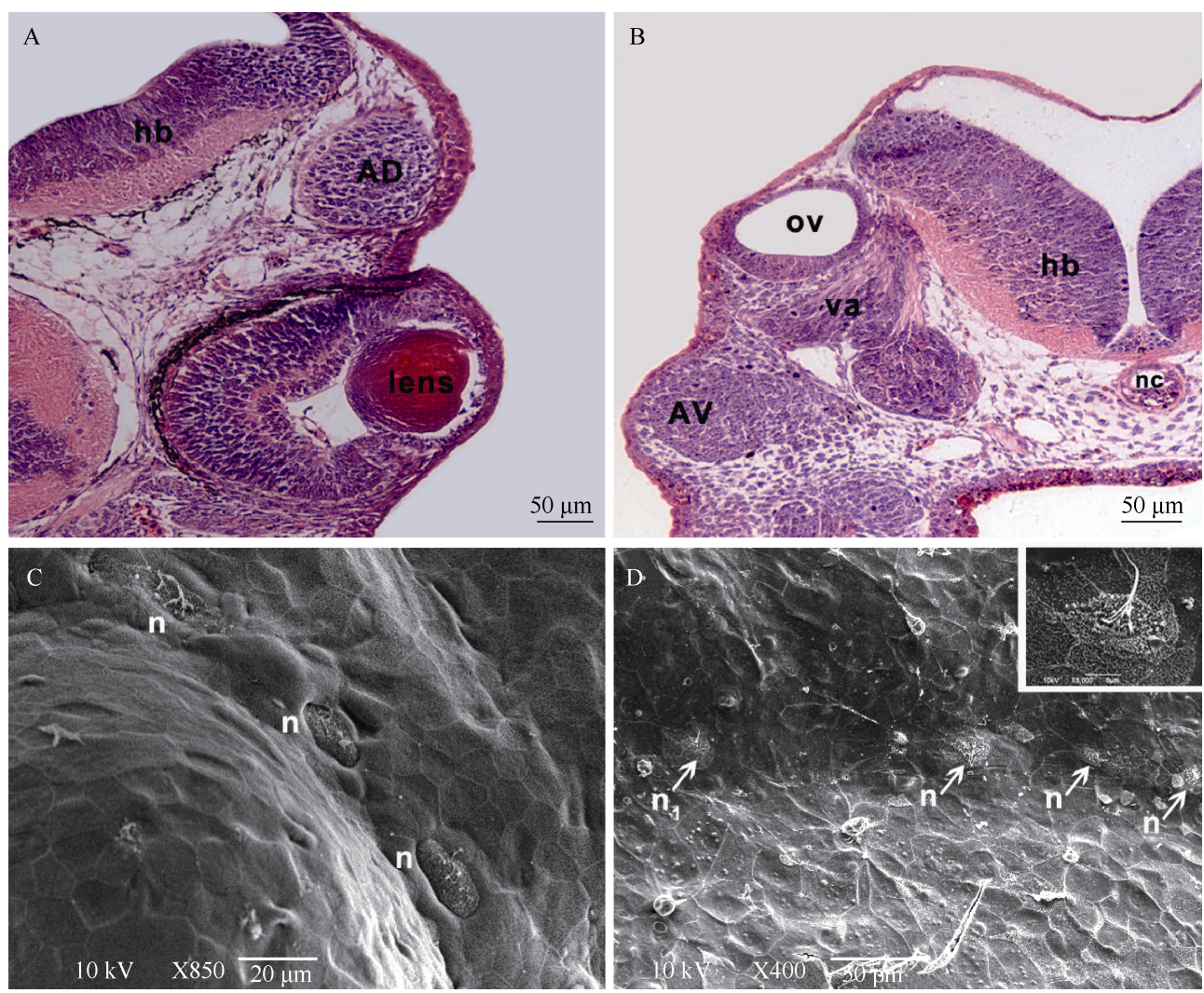

图 2 西伯利亚鲟头部横切及相关侧线发育模式图

Fig.2 Photomicrographs of transverse sections through the head and the scanning electron micrographs of erupting neuromasts located to the otic lateral line

A, B) 1 日龄西伯利亚鲟头部横切图; C) 1 日龄听侧线神经丘扫描电镜图; D) 5 日龄后侧线基板向躯干部迁移, 在躯干部特定位置遗留下部分细胞, 发 育成神经丘。内插图为神经丘 $\left(\mathrm{n}_{1}\right)$ 放大图。 $\mathrm{AD}$ : 前背侧基板神经节; $\mathrm{AV}$ : 前腹侧基板神经节; lens: 晶状体; $\mathrm{hb}$ : 后脑; va: 听神经中枢; $\mathrm{nc}$ : 脊索; ov: 听囊; $\mathrm{n}$ : 神经丘。

A, B) day 1 post hatching; C) scanning electron micrographs of erupting neuromasts located to the otic lateral line at day 1 post hatching; D) the posterior lateral line placode actively migrates along the trunk, depositing neuromasts at intervals.. Insert: Higher magnification of neuromast ( $\mathrm{n}_{1}$ ). AD: anterodorsal placode ganglion; AV: anteroventral placode ganglion; hb: hindbrain; va: vestibuloacoustic ganglion; nc: notochord; ov: otic vesicle; n: neuromast.

\section{2 侧线感受器的发育}

2.2.1 神经丘的发育 扫描电镜下, 0 日龄, 神经丘 在听侧线基板感觉嵴中出现，呈椭圆形，外层为套 细胞, 形成椭圆形带, 中央可见 2 3 束感觉毛细胞 的纤毛延伸突出于神经丘表面(图 3A)。5 日龄, 神 经丘呈椭圆形凹陷，凹陷周围由套细胞的延伸组成， 感觉毛细胞的纤毛束数量增多, 并且延长突向表面, 因黏液的分泌使纤毛黏成多束(图 3B)。29日龄, 神 经丘表面胶质覆盖物形成, 毛细胞和支持细胞继续 生长, 毛细胞和支持细胞的数量也不断增加。49日 龄, 神经丘呈长椭圆形, 长径约 $47 \mu \mathrm{m}$, 短径约 20 $\mu \mathrm{m}$; 神经丘中央形成由约 22 个毛细胞组成的感觉 极性区域, 每一个毛细胞均有一根动纤毛和数根静 纤毛, 动纤毛总是位于细胞的一侧, 静纤毛群侧位 于另一侧且排列成排, 使得细胞具有极性, 其周围
由大量的支持细胞和套细胞组成(图 3C)。此时, 部 分神经丘正被管道覆盖(图 3D)。52 日龄, 神经丘已 完全被骨化的管道所包埋, 并且在侧线管内有规律 地按一定距离分布。

2.2.2 壸腹器官的发育 7 日龄, 感觉上皮开始下 陷，含少量感觉细胞和支持细胞，形状不规则，染 色较浅 (图 4A)。9 日龄, 感觉上皮下陷明显, 感觉 细胞和支持细胞数量增加, 感觉细胞核染色较深 (图 4B)。29 日龄, 壸腹器官在表皮开口的宽度以及 下陷的深度明显增加, 感觉细胞和支持细胞数量继 续增加。在吻部腹面两侧可见少数个别的壸腹器官 表皮细胞覆盖壶腹器官中央区域留下 $3 \sim 4$ 个小的 开口(图 5)。36 日龄, 感觉上皮继续下陷, 感觉细胞 数量较支持细胞多, 排列紧密(图 4C)。57 日龄, 大 部分壶腹器官发育成熟, 呈长颈瓶状, 瓶结构内部 

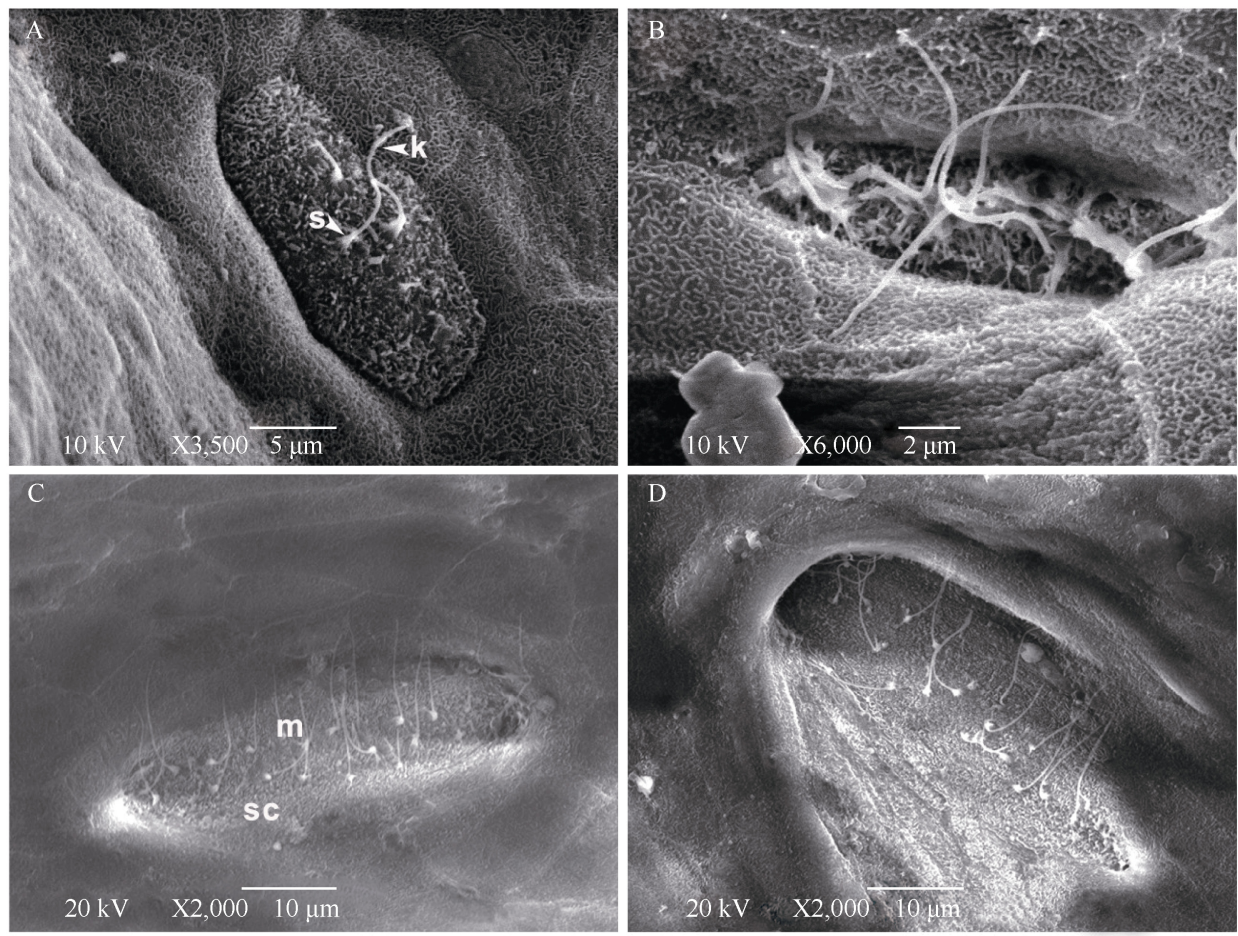

图 3 西伯利亚鲟头部神经丘发育图

Fig.3 Scanning electron micrographs of various stages in the development of neuromasts of the head A) 0 日龄管道神经; B) 5 日龄管道神经; C, D) 49 日龄管道神经。 K: 动纤毛; S: 静纤毛; $\mathrm{m}$ : 神经丘感觉极性区域; SC: 支持细胞。

A) the neuromast at the hatching stage; B) the neuromast at day 5 post hatching; C, D) the neuromast at day 49 post hatching. k: kinocilium; s: stereocilia; m: sensory macula of neuromast; sc: support cells。
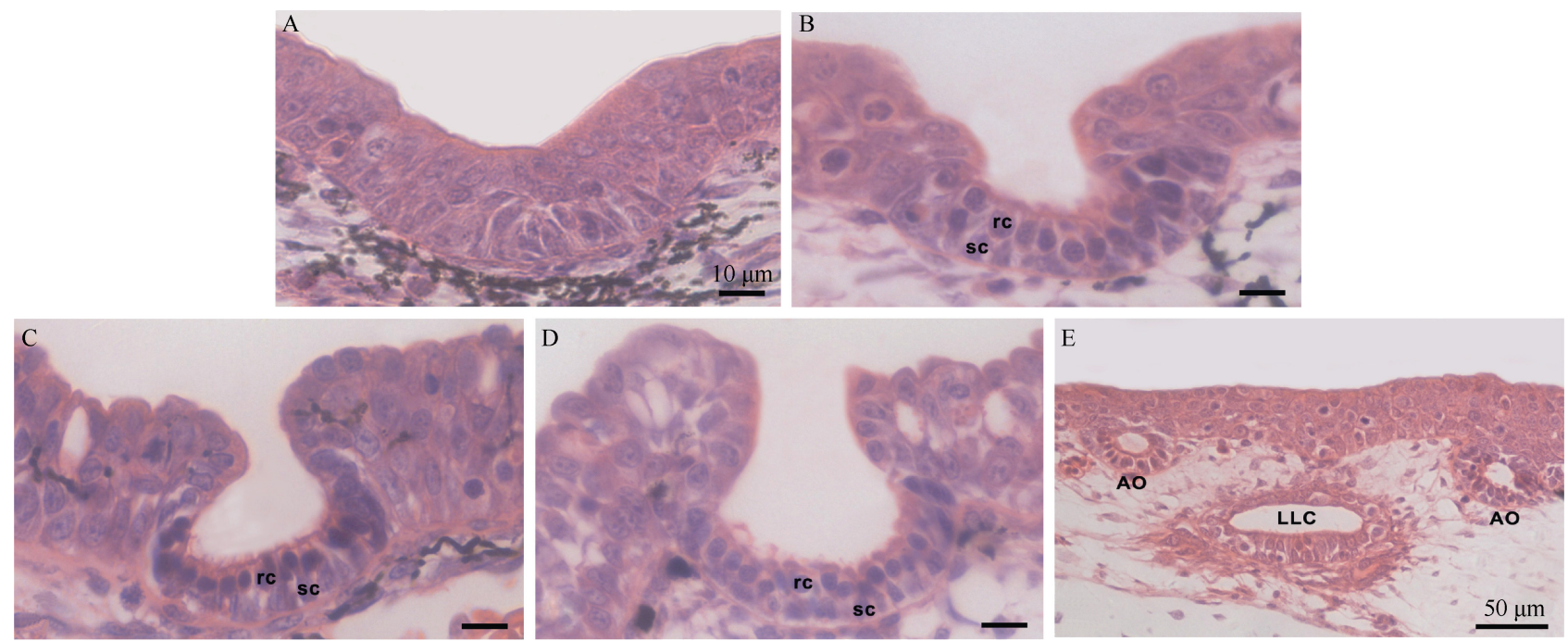

图 4 壸腹器官发育图

Fig.4 Micrographs under optical microscope of ampullary organs at different development stages

A) 7 日龄壸腹器官; B) 9 日龄壸腹器官; C) 36 日龄壸腹器官; D) 57 日龄壶腹器官; E) 57 日龄, 可见大多数壸腹器官常分布在神经丘两侧。 rc: 感觉细 胞; sc：支持细胞; AO：壸腹器官; LLC：侧线管。

A) ampullary organ at day 7 post hatching; B) ampullary organ at day 9 post hatching; C) ampullary organ at day 36 post hatching; D) ampullary organ at day 57 post hatching; E) the distribution of ampullary organs are closely associated with the neuromast lines at day 57 post hatching. rc: receptor cells; sc: support cells; AO: ampullary organ; LLC: lateral line canal.

充满黏液，瓶口开口于皮肤表皮，与外环境相接触， 瓶的底部埋在真皮层内，感觉细胞和支持细胞位于
瓶底内部，感觉细胞呈卵圆形，围腔排列，球形的 细胞核染色较深; 支持细胞位于底部基膜之上, 细 

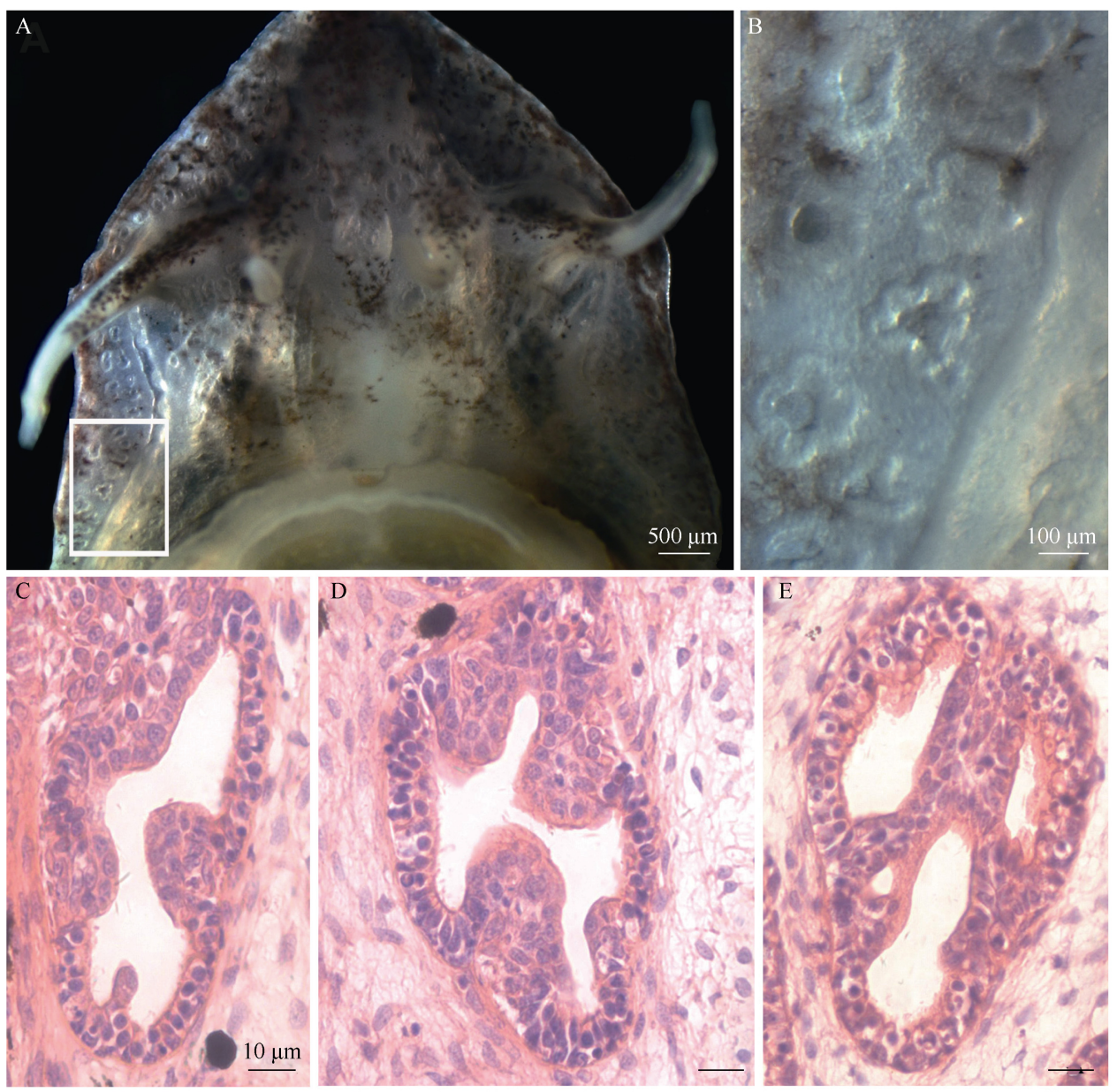

图 5 吻部腹面壶腹器官表皮细胞向壶腹器官中央区域覆盖示意图

Fig.5 Micrographs of the epidermal cells surrounded some single openings of the ampullary organs at the ventral surface of the head migrating toward the central area of the ampullary organs

A) 29 日龄头部腹面; B) A 中白色方框放大图; C, D, E) 29 日龄, 壶腹器官横切图。

A) the ventral surface of the head at day 29 post hatching; B) higher magnification of ampullae shown in white frame of A; C, D, E) a horizontal section through an ampullary organ at 29 post hatching.

胞质延伸通向腔内，将感觉细胞隔开(图 4D)。大部 分壸腹器官分布在神经丘两侧(图 4E), 也有单侧分 布，例如它们只单侧存在于听侧线管背部。鳃盖处 壶腹器官除外，鰓盖处未发现神经丘的存在。

扫描电镜下, 7 日龄, 壶腹器官原基已在前背侧 基板、前腹侧基板、听基板、横枕基板中形成(图 6A)。9 日龄, 壶腹器官主要分布在吻部腹面两侧(图 $6 \mathrm{~B})$, 并且出现单个发育完善的壸腹器官, 吻部两 侧及背面、鰓盖等处陆续有壶腹器官原基形成。 15 日龄, 吻部腹面中央壸腹器官表皮开口增多(图 6C), 从开口处可见管内有大量的黏液物质存在。有少量 壶腹器官在吻部两侧及背面、鳃盖等处出现。29日 龄, 吉腹器官布满整个吻部腹面(图 6D), 从壸腹器 官表皮开口可见管底有大量微线毛分布(图 6E)。36 日龄, 吻部腹面开始出现多个壶腹器官聚集成簇,
初具“梅花状”。57 日龄，吻部腹面可见 3 4 个壶腹 器官聚集在一起, 呈“梅花状”, 分布紧密, 并且该 部分皮肤表面凹陷，形成花朵状凹穴(图 6F)。

\section{3 侧线管道的形成}

头部侧线管道的形成。9 日龄，管道神经丘全 部出现在皮肤表面，神经丘及神经丘之间的表皮略 有凹陷，侧线管道开始形成(图 7A)。15 日龄，管道 上皮继续下陷，呈沟状，形成开放式管道(图 7B)。 36 日龄, 管道下陷明显, 周围有软骨组织生成(图 7C)。49 日龄，管道底部变宽，神经丘位于底部中间， 管道上方开始闭合(图 7D)。52 日龄，侧线管道完全 封闭, 并被软骨包围, 近圆形, 周围有少量黑色色 素分布(图 7E)。眶上管、听管、颌管、横枕管形成 较眶下管略早些。随着仔鱼的生长, 侧线管道直径 不断增大。 

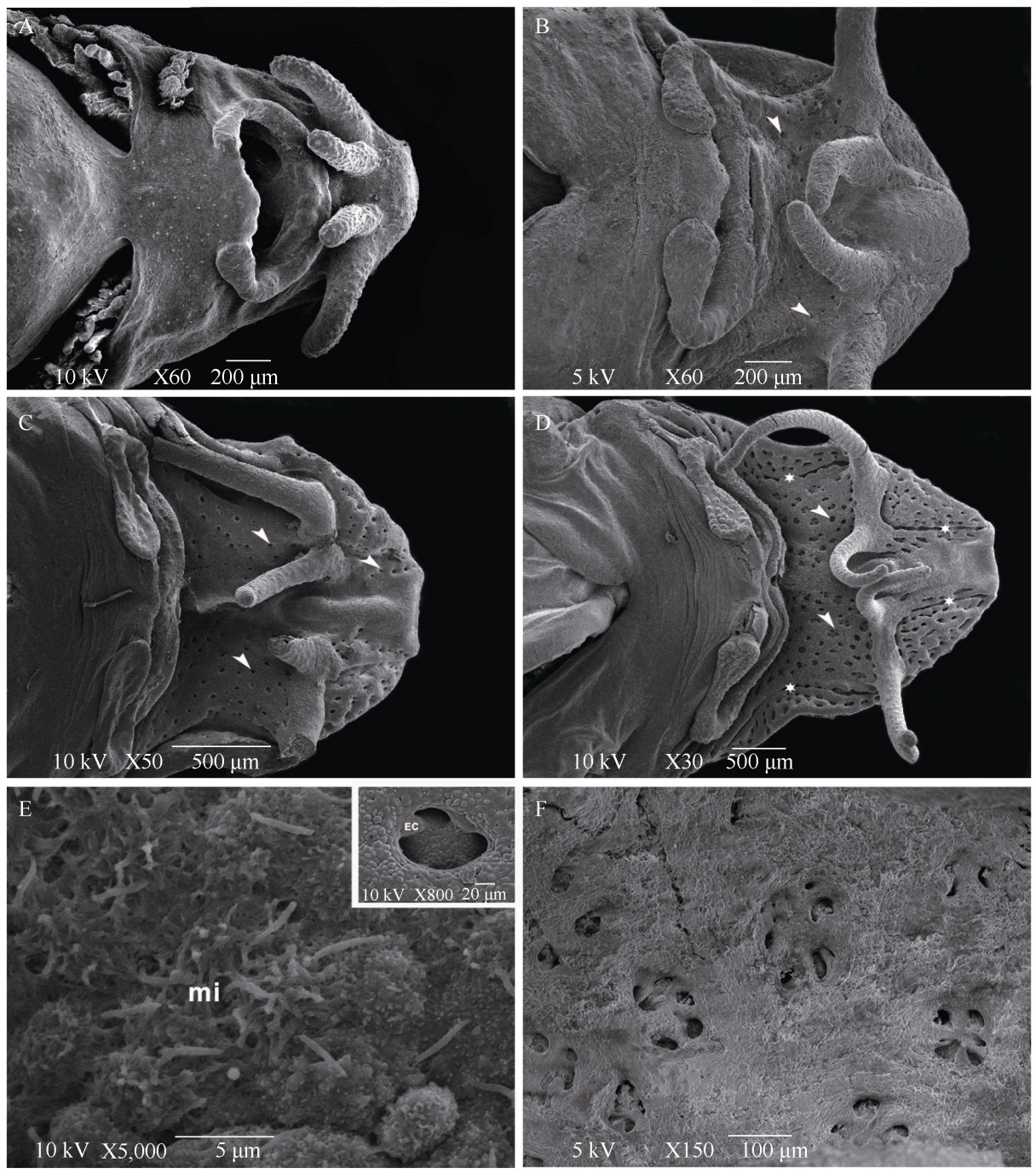

图 6 头部腹面壸腹器官发育图

Fig.6 Scanning electron micrographs of various stages in the development of ampullary organs on the ventral rostrum A) 7 日龄头部腹面; B) 9 日龄头部腹面, 箭头, 壸腹器官; C) 15 日龄头部腹面, 箭头, 壸腹器官; D) 29 日龄头部腹面, 箭头, 壸腹器官, *: 侧线管; E) 29 日龄壸腹底部存在大量的微线毛, 内插图为壸腹开口低倍放大图; F) 57 日龄, 吻部腹面可见 $3 \sim 4$ 个壸腹器官聚集在一起。 mi: 微线毛。

A) the ventral surface of the head at day 7 post hatching; B) the ventral surface of the head at day 9 post hatching. Arrowhead, ampullary organs; C) the ventral surface of the head at day 15 post hatching. Arrowhead, ampullary organs; D) the ventral surface of the head at day 29 post hatching. Arrowhead, ampullary organs; asterisk, lateral line canal; E) high magnification showing the clusters of microvilli covering the floor of the ampulla at day 29 post hatching. Insert: A low power scaning electron micrograph looking down into an ampulla, revealing clusters of microvilli on the floor of the ampulla. F) the ampullary organs are arranged in clusters of 3-4 at the ventral surface of the head at day 57 post hatching. mi: microvilli.

躯干侧线管道与头部管道发育相似(图 8), 躯 干侧线管道直径比头部要小。57 日龄，侧骨板发育 完全，身干侧线管道己完全埋于侧骨板中，并且被 软骨包围(图 8E), 在每一骨板鳞片上均有一小孔与 外界相连。

\section{3 讨 论}

从胚胎发育的观点看, 神经系统的组成成分主 要来源于神经胚的两部分: 神经嵴和外胚层板。神
经嵴起源于神经管最靠背部的区域，能迁移至身体 不同部位，产生各种类型分化细胞，主要包括感觉 交感和副交感神经系统的神经元和胶质细胞、肾上 腺的髓质细胞、表皮中的黑色素细胞，以及头部的 骨骼和结缔组织成分(Baker，2008; Dupin et al, $2007)$; 外胚层板是由胚胎头部特定区域的外胚层 增厚单独形成的, 能发育形成各种感觉细胞及其神 经节，主要包括嗅基板、听基板、三叉基板、深基 板及侧线基板等(Baker et al, 2008; Schlosser, 2006, 

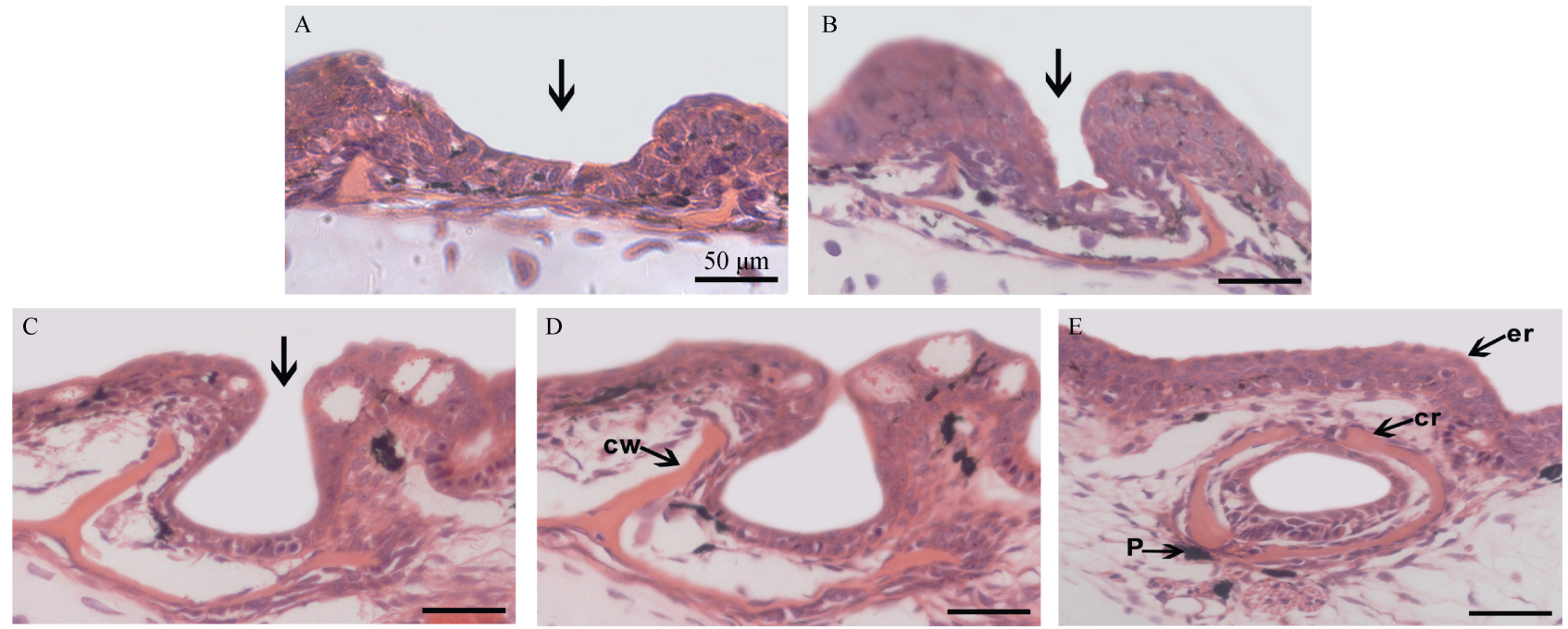

图 7 头部侧线管道发育图

Fig.7 Micrographs under optical microscope of lateral line canal of head at different development stages A) 9 日龄管道纵切; B) 15 日龄管道纵切; C) 36 日龄管道纵切; D) 49 日龄管道纵切; E) 52 日龄管道纵切。cw: 骨化的管道壁; er: 上皮细胞顶; cr: 固 化的管道顶部; P: 色素。

A) a vertical section through the canal at day 9 post hatching; B) a vertical section through the canal at day 15 post hatching; C) a vertical section through the canal at day 36 post hatching; D) a vertical section through the canal at day 49 post hatching; E) a vertical section through the canal at day 52 post hatching. cw: ossified canal walls; er: epithelial roof; cr: ossified canal roof; P: pigments.
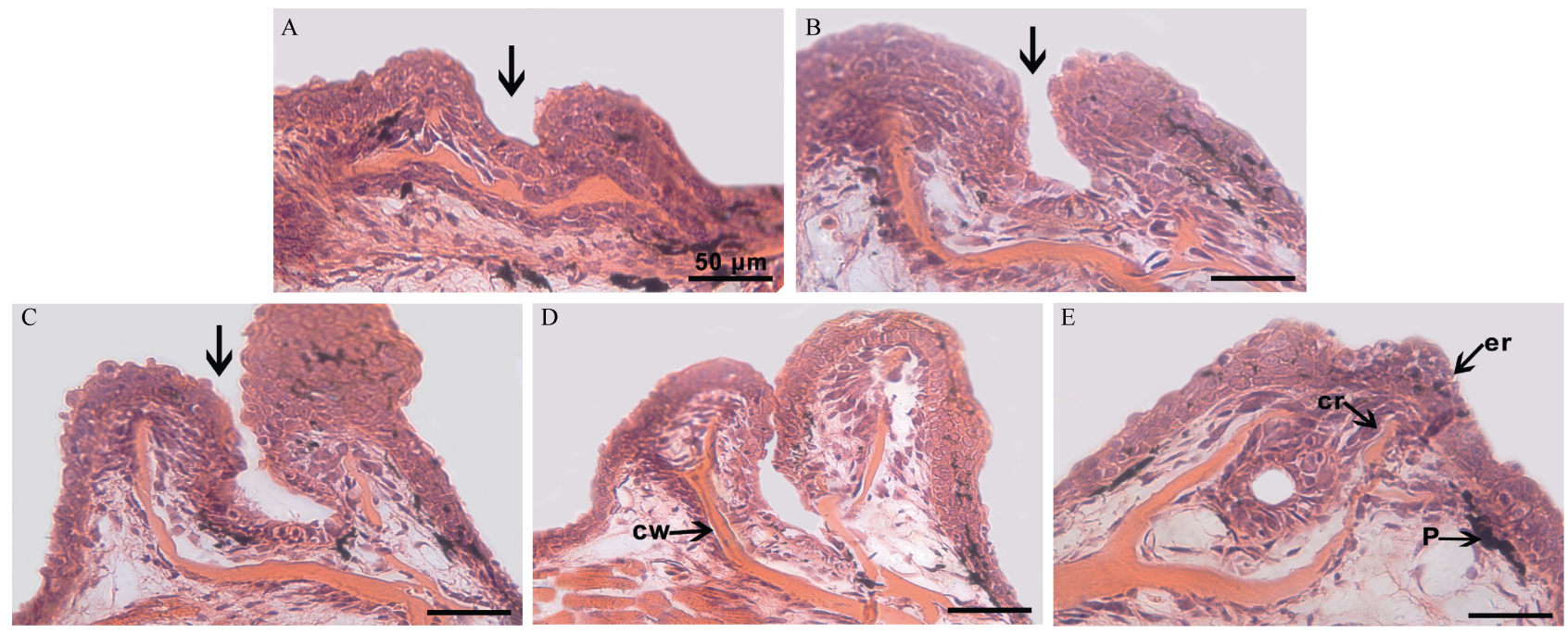

图 8 躯侧线管道发育图

Fig.8 Micrographs under optical microscope of lateral line canal of trunk at different development stages

A) 9 日龄管道纵切; B) 15 日龄管道纵切; C) 36 日龄管道纵切; D) 49 日龄管道纵切; E) 57 日龄管道纵切。 cw: 骨化的管道壁; er: 上皮细胞顶; cr: 固化的管道顶部; P: 色素。

A) a vertical section through the canal at day 9 post hatching; B) a vertical section through the canal at day 15 post hatching; C) a vertical section through the canal at day 36 post hatching; D) a vertical section through the canal at day 49 post hatching; E) a vertical section through the canal at day 57 post hatching. cw: ossified canal walls; er: epithelial roof; cr: ossified canal roof; P: pigments.

2008)。Northcutt et al (1995)运用组织嫁接和组织切 除等实验手段，证实蝾螈侧线系统由侧线基板发育 而来; 侧线基板分布在在耳部前后外肧层增厚区域; 神经丘在侧线基板的中央区域形成, 壸腹器官在侧
线基板的两侧区域形成。O'Neill et al (2007)从分子 生物学方面在小点猫鲨(Scyliorhinus canicula)中, 证明转录辅助因子 Eya4 能在侧线系统发育的整个 过程中表达，包括在所有的侧线基板中和感觉崉阶 
段; 而壸腹器官是在感觉崉阶段形成, 证明了鲨鱼 的电感受器起源于侧线基板, 并且发现转录因子 $T b x 3$ 是鉴鱼侧线神经系统的特定标记。本文研究表 明, 西伯利亚鲟仔鱼听囊前后外胚层增厚区域存在 6 对侧线基板，除后侧线基板细胞向躯干迁移外, 其他侧线基板都形成一短暂性存在的感觉崉结构; 神经丘在感觉嵴的中央区域形成, 壸腹器官在感觉 嵴的两侧形成; 壸腹器官的发育比神经丘晚一周左 右，这与蝾螈侧线发育模式相似(Northcutt et al, 1994, 1995)。目前有学者通过研究认为神经嵴细胞 也参与的侧线系统的发育, 比如 Collazo et al (1994) 运用胚胎预定命运图绘制技术，研究表明斑马鱼、 条鰙鱼、蝾螈侧线系统具有双重起源, 既起源于外 胚层板, 又起源于神经崉, 但是由于在神经胚期神 经嵴细胞和外胚层板细胞混合在一起，通过向胚胎 注射 DiI 难以区分细胞的种类。基于电感受器中基 因 Sox8 和 HNK1 抗体的交叉反应, Freitas et al (2006) 认为鲨鱼的电感受器起源于神经焨细胞, 但是该实 验所用都不是神经嵴细胞特定的标记物。本研究通 过组织学和扫描电镜观察, 为西伯利亚鲟侧线发育 的研究提供形态学方面证据。为进一步认识侧线发 育过程, 需要运用分子生物学和细胞生物学等手段, 对侧线发育相关功能基因表达及细胞迁移等进行 研究。

前腹侧基板一般能发育形成前鳃盖下领管。 密西西比铲鲟的前腹侧基板虽没有形成管道结构,

\section{参考文献:}

Baker CVH. 2008. The evolution and elaboration of vertebrate neural crest cells[J]. Curr Opin Genet Dev, 18(6): 536-543.

Baker CVH, Bronner-Fraser M. 2001. Vertebrate cranial placodes I. Embryonic induction[J]. Dev Biol, 232(1): 1-61.

Baker CVH, O'Neill P, McCole RB. 2008. Lateral line, otic and epibranchial placodes: Developmental and evolutionary links?[J]. J Exp Zool B: Mol Dev Evol, 310(4): 370-383.

Camacho S, Del Valle Ostos M, Llorente JI, Sanz A, García M, Domezain A, Carmona R. 2007. Structural characteristics and development of ampullary organs in Acipenser naccarii $[\mathrm{J}]$. Anat Rec, 290(9): 1178-1189.

Cheng H, Huang SQ, Heatwole H. 1995. Ampullary organs, pit organs, and neuromasts of the Chinese giant salamander, Andrias davidianus $[\mathrm{J}] . J$ Morphol, 226(2): 149-157.

Collazo A, Fraser SE, Mabee PM. 1994. A dual embryonic origin for vertebrate mechanoreceptors[J]. Science, 264(5157): 426-430.

Dupin E, Calloni G, Real C, Gonçalves-Trentin A, Le Douarin NM. 2007. Neural crest progenitors and stem cells[J]. C R Biol, 330(6-7): 521-529.

Freitas R, Zhang GJ, Albert JS, Evans DH, Cohn MJ. 2006. Developmental origin of shark electrosensory organs[J]. Evol Dev, 8(1): 74-80.
但在鳃盖处出现 5 个神经丘(Gibbs \& Northcutt, 2004)。在西伯利亚鲟中前腹侧基板仅发现有少量 的壶腹器官，没有神经丘的结构出现。壶腹器官一 般在神经丘的两侧都有分布(Northcutt et al, 1994), 在西伯利亚鲟及其他鲟科鱼类中，听侧线管的腹侧 没有売腹器官分布，仅在其背侧有壸腹器官分布 (Gibbs \& Northcutt, 2004)。这些发育特点差异性可 能与个体及其生存环境适应性有关，也可能是侧线 系统进化的表现。

29 日龄，在吻部腹面两侧可见少数个别的壶腹 器官表皮细胞覆盖壶腹器官中央区域留下 3４ 个 小的开口。壸腹器官表皮细胞这种特殊的迁移尚未 在国内外相关研究文献报道。壸腹管内可见大量的 微线毛存在, 在其他鲟形目鱼类、多魦鱼、软骨鱼 类中也存在类似的结构(Camacho et al, 2007)。

在我国, 虽然西伯利亚鲟已突破了全季节全人 工催产和人工受精技术(Zhuang et al, 2009), 但是仔 鱼培育阶段必要时候仍需要使用加热装置进行控 温, 一般在小水体中常使用加热棒控温, 加热棒一 般分为铜加热棒和玻璃加热棒两种, 由于鲟鱼类壸 腹器官能检测水环境下周边微弱电流, 对金属极为 敏感，产生逃避行为(Gurgens et al, 2000; Teeter et al, 1980)。西伯利亚鲟仔鱼在 9 日龄初次摄食阶段已有 壶腹器官发育成熟, 用铜加热棒可能会使西伯利亚 鲟出现慌乱逃鼌行为, 影响其摄食及正常生长, 并 导致大批量死亡。

Gibbs MA. 2004. Lateral line receptors: Where do they come from developmentally and where is our research going?[J]. Brain Behav Evol, 64(3): 163-181.

Gibbs MA, Northcutt RG. 2004. Development of the lateral line system in the shovelnose sturgeon[J]. Brain Behav Evol, 64(2): 70-84.

Gisbert E, Ruban GI. 2003. Ontogenetic behavior of Siberian sturgeon, Acipenser baerii: A synthesis between laboratory tests and field data[J]. Environ Biol Fish, 67(3): 311-319.

Gisbert E, Williot P. 2002. Advances in the larval rearing of Siberian sturgeon[J]. J Fish Biol, 60(5): 1071-1092.

Gisbert E, Williot P, Castelló-Orvay F. 1999. Behavioural modifications in the early life stages of Siberian sturgeon (Acipenser baerii, Brandt)[J]. $J$ Appl Ichthyol, 15(4-5): 237-242.

Goulet J, Engelmann J, Chagnaud BP, Franosch JMP, Suttner MD, Van Hemmen JL. 2008. Object localization through the lateral line system of fish: theory and experiment[J]. J Comp Physiol A, 194(1): 1-17.

Gurgens C, Russell DF, Wilkens LA. 2000. Electrosensory avoidance of metal obstacles by the paddlefish[J]. J Fish Biol, 57(2): 277-290.

Hofmann MH, Wojtenek W, Wilkens LA. 2002. Central Organization of the Electrosensory System in the Paddlefish (Polyodon spathula)[J]. $J$ 
Comp Neurol, 446(1): 25-36.

Lin X. 2003. Preliminary study on growth characteristics of juvenile Acipenser Baeri[J]. J Putian Univ, 10(3): 30-33. [林星. 2003. 西伯利 亚鲟稚幼鱼生长特征的初步研究. 苯田学院学报, 10(3): 30-33.]

Ma J. 2007. Studies on the Early Development and Growth Pattern of Chinese Sturgeon, Acipenser sinensis, and Amur sturgeon, Acipenser schrenckii[D]. Master thesis, Shanghai Fisheries University, Shanghai. [马境. 2007. 中华鲟和施氏鲟胚后发育及生长研究[D]. 硕士学位 论文, 上海水产大学, 上海.]

Mogdans J, Geisen S. 2009. Responses of the goldfish head lateral line to moving objects[J]. J Comp Physiol A, 195(2): 151-165.

Northcutt RG. 1992. Distribution and innervation of lateral line organs in the axolotl[J]. J Comp Neurol, 325(1): 95-123.

Northcutt RG, Brändle K, Fritzsch B. 1995. Electroreceptors and mechanosensory lateral line organs arise from single placodes in axolotls[J]. Dev Biol, 168(2): 358-373.

Northcutt RG, Catania KC, Criley BB. 1994. Development of lateral line organs in the axolot1[J]. J Comp Neurol, 340(4): 480-514.

O'Neill P, McCole RB, Baker CVH. 2007. A molecular analysis of neurogenic placode and cranial sensory ganglion development in the shark, Scyliorhinus canicula[J]. Dev Biol, 304(1): 156-181.

Rodríguez A, Gisbert E. 2002. Eye development and the role of vision during Siberian sturgeon early ontogeny[J]. J Appl Ichthyol, 18(4-6): 280-285.

Schlosser G. 2006. Induction and specification of cranial placodes[J]. Dev Biol, 294(2): 303-351.
Schlosser G. 2008. Do vertebrate neural crest and cranial placodes have a common evolutionary origin?[J]. BioEssays, 30(7): 659-672.

Song JK, Yan HY, Popper AN. 1995. Damage and recovery of hair cells in fish canal (but not superficial) neuromasts after gentamicin exposure[J] Hearing Res, 91(1-2): 63-71.

Song W, Song JK, Fan CX, Zhang T, Wang B. 2010. Studies on the early embryonic development of artificially-bred Siberian sturgeon (Acipenser baeri)[J]. J fish Chn, 34(5): 777-785. [宋炜，宋佳坤，范纯 新, 张涛, 王斌. 2010. 全人工繁殖西伯利亚鲟的早期胚胎发育. 水 产学报, 34(5): 777-785.]

Teeter JH, Sznmier RB, Bennett MVL. 1980. Ampullary electroreceptors in the sturgeon Scaphirhynchus platorynchus (Rafinesque)[J]. J Comp Physiol, 138(3): 213-223.

Wang W, Zhu H, Hu HX, Tian ZH, Dong Y. 2009. Heteroplasmy in mtDNA control region and phylogenetics of five sturgeon[J]. Zool Res, 30(5): 487-696. [王巍, 朱华, 胡红霞, 田照辉, 董颖. 2009. 五种鲟 鱼线粒体控制区异质性和系统发育分析. 动物学研究, 30(5): 487-496.]

Wilkens LA, Hofmann MH. 2007. The paddlefish rostrum as an electrosensory organ: A novel adaptation for plankton feeding[J]. Bioscience, 57(5): 399-407.

Zhuang P, Song C, Zhang LZ, Zhang T, Huang XR, Wang B. 2009. Allometric growth of artificial bred Siberian sturgeon Acipenser baeri larvae and juveniles[J]. Chn J Ecol, 28(4): 681-687. [庄平, 宋超, 章 龙珍, 张涛, 黄晓荣, 王斌. 2009. 全人工繁殖西伯利亚鲟仔稚鱼发 育的异速生长. 生态学杂志, 28(4): 681-687.]

\section{昆明生物多样性大型仪器区域中心服务项目简介}

昆明生物多样性大型仪器区域中心（区域中心）是根据《中国科学院 “十一五”科研装备建设规划》部署，经中国科学院批准建设，是先期建 设的十个大型仪器区域中心之一, 也是三个生物类大型仪器区域中心之一 (北京、上海、昆明)。“区域中心” 由昆明动物研究所、昆明植物研究 所和西双版纳热带植物园联合组建。其宗旨是：整合大型仪器资源，建立有效支撑生物多样性基础研究及生物资源可持续利用研究的大型仪器共享 平台, 实现区域大型仪器的合理布局、规范管理、高效使用。“中心” 的目标是建成国际一流的具有区域特色的生物多样性大型仪器中心，使其成 为国家创新体系技术支撑平台的一个重要组成部分, 为国家在战略生物资源的创新性研究和生物产业化提供关键技术支撑。

截至 2012 年 3 月，该服务中心在用和在建的大型仪器设备共有 50 多台 (套)，今后每年将持续投入资金建设，目前设备已通过中国科学院共 享网络管理系统对外进行预约服务。具体平台和服务项目如下:

\section{一、特色基因分析平台}

1. DNA 序列分析仪 (Applied Biosystems 3730x1 DNA Analyzer)。服务项目： DNA 测序。

2. 罗氏/454 高通量测序系统（Roche/454 Genome Sequencer FLX System）。服务项目：基因组测序，转录组测序，宏基因组测序，扩增子测序等。

3. I1lumina 高通量基因组测序系统（I1lumina Hiseg 2000 Sequencing System)。服务项目：基因组 de novo 测序，基因组重测序，转录组测 序，小 RNA 测序，宏基因组测序，扩增子测序，ChIP-Seq 测序，甲基化测序等。

4. 自动化液体工作站 (Hamilton Microlab Star) 。服务项目：大规模样品的核酸提取。

\section{二、蛋白质及细胞生物学技术分析平台}

1. 质谱仪 (Bruker Autoflex speed TOF/TOF MALDI-TOF system)。服务项目: 蛋白分子量测定, 肽指纹图谱（一级质谱）二级质谱。

2. 蛋白自动测序仪 (PPSQ-31A Automated Protein/Peptide Sequencers) 。服务项目: 蛋白多肽 N 末端序列测定。

3. 流式细胞仪 (Flow Cytometer)。服务项目：分析细胞表面标志；分析细胞内抗原物质；分析细胞受体；分析细胞的 DNA 含量、细胞周 期; 分析免疫细胞的功能; 细胞分选等。

4. 激光共聚焦扫描显微镜 (Confocal Laser Scanning Microscope, CLSM)。服务项目: 细胞形态学分析（观察细胞或组织内部微细结构, 如: 细胞内线粒体、内质网、高尔基体、微管、微丝、细胞桥、染色体等亚细胞结构的形态特征; 半定量免疫苂光分析); 苂光原位杂交研究; 基因定位 研究及三维重建分析。

5. 液氮原 (Liquid Nitrogen Pump)。服务项目: 供应液氮。 